$06 ; 13$

\title{
Планарные емкостные структуры на основе сегнетоэлектрических пленок титаната-станната бария на сапфире для сверхвысокочастотных применений
}

\author{
(C) А.В. Тумаркин, М.В. Злыгостов, А.Г. Гагарин, А.Г. Алтынников, Е.Н. Сапего
}

Санкт-Петербургский государственный электротехнический университет „ЛЭТИ“, Санкт-Петербург, Россия

E-mail: avtumarkin@yandex.ru

Поступило в Редакцию 7 марта 2019г.

В окончательной редакции 1 апреля 2019г.

Принято к публикации 1 апреля 2019г.

\begin{abstract}
Исследованы структурные свойства сегнетоэлектрических пленок титаната-станната бария на подложках сапфира и сверхвысокочастотные характеристики планарных емкостных элементов на их основе. Установлено, что состав газовой среды в процессе осаждения пленки оказывает существенное влияние на кристаллическую структуру, фазовый состав пленок и их электрические характеристики. Впервые продемонстрирован низкий уровень диэлектрических потерь планарных емкостных элементов на основе пленок титаната-станната бария в частотном диапазоне $2-60 \mathrm{GHz}$ при высокой управляемости.
\end{abstract}

Ключевые слова: сегнетоэлектрические пленки, титанат-станнат бария, сверхвысокочастотные применения.

DOI: 10.21883/PJTF.2019.13.47947.17776

Интерес к сегнетоэлектрикам обусловлен зависимостью диэлектрической проницаемости данных материалов от внешнего электрического поля, относительно низким уровнем диэлектрических потерь и устойчивостью к сигналу высокого уровня мощности [1,2]. Указанные свойства делают сегнетоэлектрические (СЭ) материалы перспективными для применения в таких сверхвысокочастотных (СВЧ) устройствах, как вариконды, фазовращатели, перестраиваемые фильтры [3,4]. Наиболее исследованными СЭ-материалами для СВЧ-применений являются пленочные твердые растворы титаната бария-стронция $\mathrm{Ba}_{x} \mathrm{Sr}_{1-x} \mathrm{TiO}_{3}$ (BST) $[1,5,6]$.

Как альтернативу BST можно рассматривать титанатстаннат бария $\mathrm{BaSn}_{x} \mathrm{Ti}_{1-x} \mathrm{O}_{3}$ (BSnT) [7,8]. Преимуществами данного материала являются меньшие величины диэлектрической релаксации и возможность приложения больших полей управления [9], что важно для реализации мощных сегнетоэлектрических СВЧ-устройств.

На данный момент опубликован ряд работ, в которых исследуются структурные и электрические свойства пленок BSnT с целью их применения в электрически управляемых устройствах [7-14]. Отметим, что в подавляющем большинстве работ рассматриваются твердые растворы с содержанием олова, не превышающим $30 \%$, что обеспечивает высокие значения диэлектрической проницаемости и, как следствие, сравнительно высокие потери. Характеристики слоев BSnT зависят от температуры осаждения [7-10], подложки [11,12], давления и состава рабочего газа $[13,14]$. В данных работах электрические характеристики слоев исследуются в составе плоскопараллельных емкостных структур металл-диэлектрик-металл (МДМ). Конструкция МДМ-конденсатора позволяет использовать малые управляющие напряжения для изменения емкости, но именно вследствие этого применение МДМ-структур возможно только в малосигнальных устройствах. Однако одно из главных потенциальных преимуществ СЭ-устройств перед полупроводниковыми аналогами, а именно возможность работы при высоких уровнях мощности, может быть реализовано только в планарном исполнении на диэлектрической подложке [2].

В немногочисленных работах, посвященных осаждению пленок BSnT на диэлектрик, исследуются свойства полученных слоев на структурно согласованных подложках, таких как $\mathrm{LaAlO}_{3}$ и $\mathrm{MgO}[15,16]$, не обладающих оптимальными СВЧ-свойствами. Данные подложки обеспечивают возможность ориентированного роста пленки, однако по интегральным СВЧ-характеристикам планарные структуры BSnT на этих подложках уступают, например, BST-аналогам на сапфире [17]. Кроме того, следует отметить, что в подавляющем большинстве работ приводятся экспериментальные данные по диэлектрическим потерям в емкостных структурах BSnT в частотном диапазоне $1 \mathrm{kHz}-1 \mathrm{MHz}$. Эти данные не позволяют сделать вывод о применимости полученных пленок на сверхвысоких частотах.

В связи с этим целью настоящей работы является исследование структуры и высокочастотных диэлектрических свойств тонких слоев BSnT, выращенных на подложках сапфира, с целью их дальнейшего применения в составе мощных нелинейных элементов СВЧ-диапазона.

Осаждение покрытий проводилось методом высокочастотного магнетронного распыления керамической мишени состава $\mathrm{BaSn}_{0.5} \mathrm{Ti}_{0.5} \mathrm{O}_{3}$ на подложки чистого сапфира $\left(\mathrm{Al}_{2} \mathrm{O}_{3}\right.$ (r-срез)). Выбор состава с высоким содержанием олова связан с необходимостью получения 


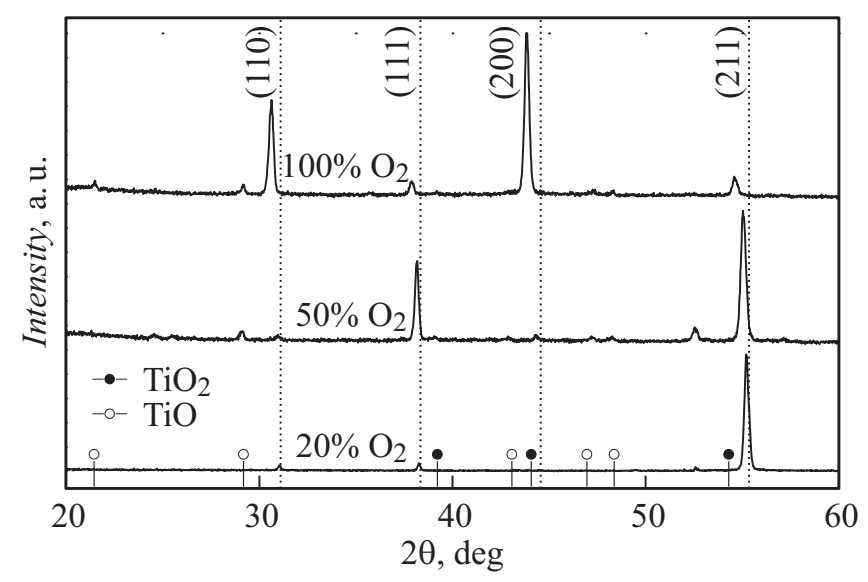

Рис. 1. Дифрактограммы тонких пленок BSnT на подложках $\mathrm{Al}_{2} \mathrm{O}_{3}$, полученных при различных концентрациях кислорода в газовой среде.

пленок с низкими значениями диэлектрических потерь на сверхвысоких частотах. Температура подложки составляла $750^{\circ} \mathrm{C}$ [7]. В качестве рабочего газа в процессе осаждения использовалась смесь аргона и кислорода в соотношениях 50/50, 80/20, а также чистый кислород; давление рабочего газа в процессе осаждения пленок составляло $2 \mathrm{~Pa}$. После осаждения пленки охлаждались в атмосфере рабочего газа со скоростью $2-3^{\circ} \mathrm{C} / \mathrm{min}$. Толщина пленок составляла $500 \mathrm{~nm}$.

Кристаллическая структура и фазовый состав полученных пленок исследовались методом рентгеновской дифракции с помощью дифрактометра ДРОН-6 на эмиссионной спектральной линии $\mathrm{Cu} K_{\alpha 1}(\lambda=1.5406 \AA)$. Для электрофизических исследований на основе пленок BSnT на сапфире были сформированы планарные конденсаторы, ширина зазора в которых составляла $5 \mu \mathrm{m}$. Верхние электроды конденсаторов были изготовлены посредством термического осаждения пленки $\mathrm{Cu}$ толщиной $1 \mu \mathrm{m}$ с адгезионным подслоем хрома с последующей литографией и химическим травлением. Измерения емкости $C$ и добротности $Q=1 / \tan \delta$ конденсаторов были проведены на частоте $2 \mathrm{GHz}$ с помощью полуволнового полоскового резонатора и векторного анализатоpa HP 8719C. Резонатор обеспечивает ненагруженную добротность 1000, погрешность измерения емкости и добротности 1 и 5\% соответственно, возможность подачи управляющего напряжения $U$ до $1000 \mathrm{~V}$, что соответствует напряженности управляющего поля $200 \mathrm{~V} / \mu \mathrm{m}$. Управляемость конденсаторов рассчитывалась как отношение емкостей при нулевом и максимальном приложенном напряжении управления: $n=C(0 \mathrm{~V}) / C\left(U_{\max }\right)$. Характеризация образцов на частоте $60 \mathrm{GHz}$ проводилась по методике открытого резонатора (Фабри-Перо).

На рис. 1 представлены дифрактограммы пленок BSnT, полученных при различных концентрациях кислорода в составе рабочего газа. Пунктирные линии соответствуют угловым положениям рефлексов для со- става распыляемой мишени $\mathrm{BaSn}_{0.5} \mathrm{Ti}_{0.5} \mathrm{O}_{3}$. Отметим, что позиции рефлексов существенно смещаются в сторону больших углов при уменьшении содержания кислорода в газовой среде, что для поликристаллических образцов означает изменение состава твердого раствора. Исходя из угловых положений рефлексов в атмосфере чистого $\mathrm{O}_{2}$ формируется поликристаллическая пленка состава $\mathrm{BaSn}_{0.96} \mathrm{Ti}_{0.04} \mathrm{O}_{3}$, что соответствует практически чистому $\mathrm{BaSnO}_{3}$. Вероятной причиной формирования станната бария может быть присутствие на подложке примесных фаз простых оксидов титана (см. положения рефлексов для оксидов титана $\mathrm{TiO}$ и $\mathrm{TiO}_{2}$ на рис. 1). Схожая ситуация при формировании твердого раствора титанатацирконата бария описывается в [18].

Иной фазовый состав демонстрируют пленки, осажденные при пониженной концентрации кислорода. В газовой смеси $\mathrm{Ar} / \mathrm{O}_{2}$ с соотношением компонентов 50/50 образуется поликристаллическая пленка BSnT состава $\mathrm{BaSn}_{0.68} \mathrm{Ti}_{0.32} \mathrm{O}_{3}$ с доминирующими ориентациями роста (111) и (211). Уменьшение содержания кислорода до 20\% приводит к росту пленки BSnT с составом $\mathrm{BaSn}_{0.58} \mathrm{Ti}_{0.42} \mathrm{O}_{3}$, близким к составу мишени, и текстурой роста (211), которая, по-видимому, может быть объяснена влиянием гексагональной структуры подложки сапфиpa $r$-среза. Дальнейшее уменьшение концентрации кислорода в газовой среде может привести к возникновению дефицита по кислороду в пленке [14] и представляется нецелесообразным.

На рис. 2 показано изменение углового положения рефлекса (211) для исследуемых образцов BSnT при изменении концентрации кислорода в газовой среде. Пунктирными линиями обозначены положения рефлекса (211) для чистого $\mathrm{BaSnO}_{3}$ (BSO) и для состава распыляемой мишени (BSnT 5/5). Сдвиг рефлекса показывает изменение параметра элементарной ячейки, который уменьшается от 4.11 до $4.07 \AA$ при снижении содержания кислорода.

Таким образом, согласно данным рис. 1 и 2, а также данным [18], формирование твердых растворов с

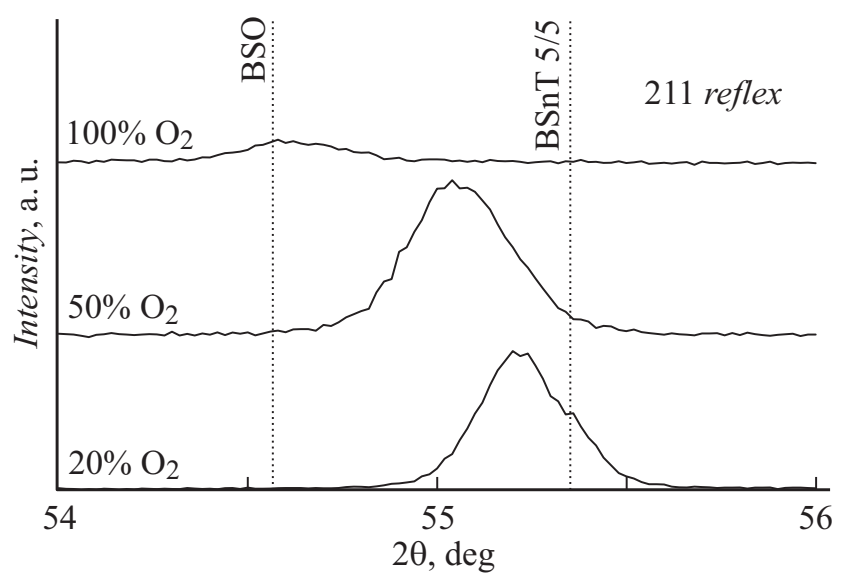

Рис. 2. Угловое положение рефлекса (211) для исследуемых образцов BSnT. 
Сравнительные электрические характеристики планарных структур BSnT

\begin{tabular}{|c|c|c|c|c|c|}
\hline Подложка & Состав & Управляемость & $\begin{array}{l}\tan \delta \\
(0 \mathrm{~V})\end{array}$ & $\begin{array}{c}\text { Частота, } \\
\text { MHz }\end{array}$ & $\begin{array}{c}\text { Лит. } \\
\text { ссылка }\end{array}$ \\
\hline $\begin{array}{c}\mathrm{LaAlO}_{3}(100) \\
\mathrm{LaAlO}_{3}(110) \\
\mathrm{LaAlO}_{3}(111) \\
\mathrm{LaAlO}_{3}(100) \\
\mathrm{MgO}(100) \\
\mathrm{Al}_{2} \mathrm{O}_{3}(r \text {-срез })\end{array}$ & $\begin{array}{l}\mathrm{BaSn}_{0.15} \mathrm{Ti}_{0.85} \mathrm{O}_{3} \\
\mathrm{BaSn}_{0.15} \mathrm{Ti}_{0.85} \mathrm{O}_{3} \\
\mathrm{BaSn}_{0.58} \mathrm{Ti}_{0.42} \mathrm{O}_{3}\end{array}$ & $\begin{array}{l}1.55 \\
1.69 \\
1.85 \\
1.21 \\
1.16 \\
1.7\end{array}$ & $\begin{aligned} \sim 0.03 \\
\sim 0.03 \\
\sim 0.03 \\
\sim 0.01 \\
\sim 0.012 \\
\quad 0.02 \\
0.08\end{aligned}$ & $\begin{array}{c}1 \\
2000 \\
60000\end{array}$ & $\begin{array}{c}{[16]} \\
\text { Наст. } \\
\text { paб. } \\
(80 / 20)\end{array}$ \\
\hline
\end{tabular}

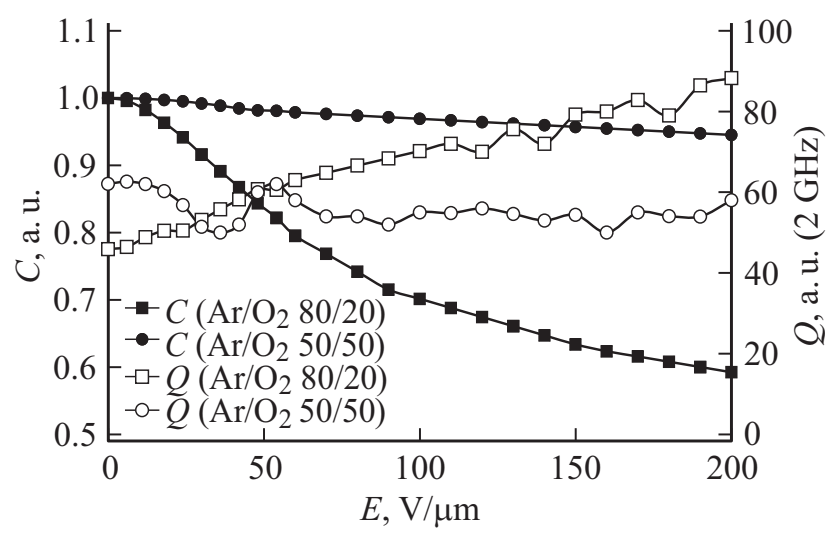

Рис. 3. Зависимость емкости и добротности планарных конденсаторов BSnT на сапфире от управляющего поля.

замещением позиции титана в ячейке перовскита на диэлектрических подложках в чисто кислородной среде затруднено, что, по-видимому, связано с наличием на подложке простых оксидов титана, скорость образования которых значительно превышает скорость формирования оксидов олова (окислительно-восстановительные потенциалы титана и олова в данном случае составляют -1.63 и $-0.14 \mathrm{~V}$ соответственно). Увеличение содержания аргона в рабочем газе приводит к подавлению процесса образования простых оксидов титана и росту концентрации олова на подложке, что обусловлено уменьшением длины термализации $\mathrm{Sn}$ при рассеянии на тяжелых атомах Ar [19]. Данные факторы обеспечивают формирование твердого раствора BSnT на сапфире.

На рис. 3 представлены зависимости емкости и добротности планарных конденсаторов, сформированных на основе пленок BSnT на сапфире. Очевидно, что в данном случае нелинейность емкостных структур будет определяться компонентным составом пленок BSnT, который в свою очередь зависит от содержания кислорода в газовой среде. Из рис. 3 видно, что максимальная управляемость $n=1.7$ достигается для пленок BSnT, осажденных при составе газовой смеси $\mathrm{Ar} / \mathrm{O}_{2}$ 80/20. При этом данные пленки проявляют высокую добротность от 45 до $90(\tan \delta$ от 0.022 до 0.011 соответственно) на частоте $2 \mathrm{GHz}$. Пленки, осажденные при повышенной концентрации кислорода, демонстрируют минимальную нелинейность, что связано с большим содержанием в твердом растворе станната бария, являющегося линейным диэлектриком. Характеристики исследуемых конденсаторов в сравнении с результатами работ $[15,16]$ представлены в таблице. Из данных таблицы следует, что полученные в настоящей работе конденсаторы проявляют перспективные для СВЧ-применений электрические характеристики на частотах вплоть до $60 \mathrm{GHz}$, что является лучшим результатом из опубликованных на данный момент для планарных емкостных структур на основе пленок BSnT.

Результаты проведенных исследований показали, что при осаждении пленок BSnT на сапфир в чистом кислороде на подложке формируется практически чистый станнат бария, что определяет отсутствие нелинейных диэлектрических свойств конденсаторов на основе данных пленок. Пленки, осажденные на сапфир в газовой смеси $\mathrm{Ar} / \mathrm{O}_{2}$, представляют собой твердый раствор $\mathrm{BaSn}_{x} \mathrm{Ti}_{1-x} \mathrm{O}_{3}$ с составом, зависящим от содержания кислорода. Для пленок BSnT на подложках $\mathrm{Al}_{2} \mathrm{O}_{3}$, осажденных в газовой среде $\mathrm{Ar} / \mathrm{O}_{2}$ с соотношением 80/20, компонентный состав, близкий к составу мишени, без включений вторичных фаз оказывает положительное влияние на их электрофизические свойства, в частности на нелинейность и уровень диэлектрических потерь. Сравнение полученных результатов с литературными данными показало, что планарные структуры BSnT на сапфире проявляют перспективные характеристики для создания на их основе управляемых СВЧ-устройств повышенного уровня рабочей мощности.

\section{Финансирование работы}

Работа выполнена при финансовой поддержке Российского научного фонда в рамках проекта № 18-79-10156 и Министерства образования и науки РФ (государственное задание № 3.3990.2017/4.6).

\section{Конфликт интересов}

Авторы заявляют, что у них нет конфликта интересов. 


\section{Список литературы}

[1] Gevorgian S. Ferroelectrics in microwave devices, circuits and systems. London: Springer, 2009. 396 p.

DOI: $10.1007 / 978-1-84882-507-9$

[2] Вендик О.Г. // ФТТ. 2009. Т. 51. В. 7. С. 1441-445. DOI: $10.1134 / \mathrm{S} 1063783409070543$

[3] Luo C., Ji J., Ling F., Li D., Yao J. // J. Alloys Compd. 2016. V. 687. P. 458-462. DOI: 10.1016/j.jallcom.2016.05.324

[4] Meyers C.J.G., Freeze C.R., Stemmer S., York R.A. // Appl. Phys. Lett. 2016. V. 109. P. 112902. DOI: 10.1063/1.4961626

[5] Song L.R., Chen Y., Wang G.S., Yang L.H., Ge J., Dong X.L., Xiang P.H., Zhang Y.Y., Tang X.D. // J. Am. Ceram. Soc. 2014. V. 97. P. $3048-3051$. DOI: $10.1111 /$ jace.13218

[6] Tumarkin A.V., Gagarin A.G., Altynnikov A.G., Gaidukov M.M., Odinets A.A., Razumov S.V., Kozyrev A.B. // Thin Solid Films. 2015. V. 593. P. 189-192.

DOI: $10.1016 /$ j.tsf.2015.09.057

[7] Tumarkin A.V., Stozharov V.M., Altynnikov A.G., Gagarin A.G., Razumov S.V., Kaptelov E.Y., Senkevich S.V., Pronin I.P., Kozyrev A.B. // Integr. Ferroelectrics. 2016. V. 173. P. $140-146$. DOI: $10.1080 / 10584587.2016 .1187055$

[8] Wu M., Zhang C., Yu S., Li L. // Ceram. Int. 2018. V. 44. P. 11466-11471. DOI: 10.1016/j.ceramint.2018.03.208

[9] Hoffmann S., Waser R.M. // Integr. Ferroelectrics. 1997. V. 17. P. $141-152$. DOI: $10.1080 / 10584589708012989$

[10] Ihlefeld J.F., Borland W.J., Maria J.-P. // J. Mater. Sci. 2008. V. 43. P. 4264-4270. DOI: 10.1007/s10853-008-2618-x

[11] Song S., Zhai J., Gao L., Yao X. // Appl. Phys. Lett. 2009. V. 94. P. 052902 . DOI: $10.1063 / 1.3073743$

[12] Gao L., Zhai J., Song S., Yao X. // Mater. Chem. Phys. 2010. V. 124. P. 192-195. DOI: 10.1016/j.matchemphys.2010.06.018

[13] Wu M., Zhang C., Yu S., Li L. // Ceram. Int. 2018. V. 44. P. 10236-10240. DOI: 10.1016/j.ceramint.2018.03.026

[14] Huang H., Wang M., Chen C., Wu N., Lin H. // J. Eur. Ceram. Soc. 2006. V. 26. P. 3211-3219.

DOI: 10.1016/j.jeurceramsoc.2005.09.109

[15] Song S., Gao L., Zhai J., Yao X., Cheng Z. // Appl. Surf. Sci. 2008. V. 254. P. $5120-5123$.

DOI: 10.1016/j.apsusc.2008.02.002

[16] Song S., Zhai J., Yao X. // Mater. Res. Bull. 2008. V. 43. P. 2374-2379. DOI: 10.1016/j.materresbull.2007.08.006

[17] Razumov S.V., Tumarkin A.V., Gaidukov M.M., Gagarin A.G., Kozyrev A.B., Vendik O.G., Ivanov A.V., Buslov O.U., Keys V.N., Sengupta L.C., Zhang X. // Appl. Phys. Lett. 2002. V. 81. P. 1675-1677. DOI: 10.1063/1.1499987

[18] Тумаркин А.В., Злыгостов М.В., Гагарин А.Г., Сапего Е.Н. // Письма в ЖТФ. 2018. Т. 44. В. 23. С. 63-70. DOI: 10.21883/PJTF.2018.23.47011.17476

[19] Тумаркин А.В., Вольплс В.А., Злыгостов М.В., Одинеи, А.A., Сапего Е.Н. // Изв. РАН. Сер. физ. 2018. Т. 82. № 3. C. 395-401. DOI: $10.7868 / \mathrm{S} 0367676518030304$ 